\title{
THE EXPONENTIAL INTEGRABILITY OF QUASI-ADDITIVE FUNCTIONALS OF GAUSSIAN VECTORS
}

UDC 519.21

\author{
V. V. BULDYGIN
}

\begin{abstract}
We study the exponential integrability of quasi-additive functionals of Gaussian random vectors.
\end{abstract}

\section{Definitions and auxiliary Results}

Let $(V, \mathcal{F})$ be a measurable vector space. This means that $V$ is a real vector space where the addition of its elements and the multiplication of its elements by real numbers agree with the $\sigma$-field $\mathcal{F}$ of subsets of the space $V$. Let $(\Omega, F, \mathrm{P})$ be a probability space.

A mapping $X$ from $\Omega$ to $V$ measurable with respect to the $\sigma$-fields $F$ and $\mathcal{F}$ is called a $V$-valued random vector. The independence of a family of $V$-valued random vectors is defined in a standard way (see [1]). A $V$-valued random vector $Y$ is called a copy of a $V$-valued random vector $X$ if $X$ and $Y$ have the same distribution.

Definition 1. A $V$-valued random vector $X$ is called Gaussian if, for each pair $\left(X_{1}, X_{2}\right)$ of two independent copies of the random vector $X$, the pair $\left(Z_{1}, Z_{2}\right)$, where

$$
Z_{1}=2^{-1 / 2}\left(X_{1}+X_{2}\right), \quad Z_{2}=2^{-1 / 2}\left(X_{1}-X_{2}\right),
$$

is also a pair of independent copies of $X$.

If $V=\mathbf{R}$ is the set of real numbers and $\mathcal{F}=B(\mathbf{R})$ is the $\sigma$-field of Borel sets, then the zero mean Gaussian random variables (and only they) satisfy Definition 1 (see, for example, [1]). This implies that for Banach spaces as well as for some general topological vector spaces $V$ Definition 1 is equivalent to the definition of a zero mean Gaussian random vector expressed in terms of the characteristic functional (see, for example, [2]).

We consider the problem on the integrability of quasi-additive functionals of $V$-valued Gaussian random vectors.

Definition 2. Let $(V, \mathcal{F})$ be a measurable vector space. A real-valued functional $g(\cdot)=$ $(g(x), x \in V)$ is called a measurable quasi-additive functional (in other words, a measurable $C$-quasi-additive functional) if

1) $g(x) \geq 0, x \in V$

2) there exists $C \geq 1$ such that $g(x+y) \leq C(g(x)+g(y))$ for $x, y \in V$;

3) the mapping $x \mapsto g(x)$ is measurable with respect to the $\sigma$-fields $\mathcal{F}$ and $B(\mathbf{R})$.

If the following additional condition holds:

4) $g(x)=g(-x), x \in V$,

then the functional $g(\cdot)$ is called a $C$-measurable quasi-additive symmetric functional.

2000 Mathematics Subject Classification. Primary 60B11, 60G15.

(C)2004 American Mathematical Society 
Remark 1. The constant $C$ involved in condition 2) is not unique. If condition 2) holds for some constant $C$, then it also holds for all $C^{\prime}>C$. The minimum constant $C_{\min }$ for which condition 2) holds can be defined as the infimum of the set of numbers $C$ satisfying that condition. This minimum constant $C_{\min }$ can be viewed as a kind of characteristic of the functional $g$. However the exact value of $C_{\text {min }}$ does not play any role in the further discussion.

Remark 2. One can consider $C$-quasi-additive functionals for $0<C<1$, too. However

$$
0 \leq \sup _{x \in V} g(x) \leq \frac{C g(0)}{1-C}<\infty
$$

for $C \in(0,1)$, since $g(x)=g(x+0) \leq C(g(x)+g(0))$, that is, the functional $g(\cdot)$ is uniformly bounded on $V$. This is a trivial case, thus we consider the case of $C \geq 1$ in condition 2).

A measurable quasi-additive functional $g(\cdot)$ is called measurable semiadditive if $C=1$. Measurable seminorms give a well-known example of semiadditive symmetric functionals. Measurable seminorms satisfy conditions 1)-4) and an extra (homogeneity) condition: $g(\lambda x)=|\lambda| g(x)$ for all $x \in V$ and $\lambda \in \mathbf{R}$. Measurable quasi-norms are a more general example of semiadditive functionals for which $g(\lambda x) \leq g(x)$ for all $x \in V$ and $\lambda \in[-1,1]$.

Simple examples of measurable $C$-quasi-additive functionals for $C>1$ are given by convex measurable functionals $(g(x), x \in V)$ such that $g(x)>0, x \neq 0$, and

$$
K=\sup _{x \neq 0}(g(2 x) / g(x)) \in(2, \infty) .
$$

In this case, $C=K / 2$. In particular, $g(\cdot)=q^{r}(\cdot)$ is a $C$-quasi-additive functional if $q(\cdot)$ is a measurable quasi-norm and $r>1$. In this case, $C=2^{r-1}>1$.

It is proved in [3, 4, 5] that for various infinite-dimensional vector spaces $V$ the following holds: if $g(\cdot)$ is a measurable seminorm and $X$ is a Gaussian random vector, then there exists $a_{0}>0$ such that $\operatorname{E} \exp \left\{a g^{2}(X)\right\}<\infty$ for all $a \in\left[0, a_{0}\right)$. The Fernique [4] proof of this result is based on Definition 1. A generalization of Fernique's method allows one to prove an analogous result for semiadditive functionals (see [6]).

The aim of this paper is to prove that there are numbers $b$ such that

$$
\mathrm{E} \exp \left\{a g^{b}(X)\right\}<\infty
$$

for every measurable $C$-quasi-additive functional $g$ of a Gaussian random vector $X$ and to show how $b$ depends on the constant $C$.

\section{MAIN RESULTS}

The first result concerns a general $C$-quasi-additive functional.

Theorem 1. Let $(V, \mathcal{F})$ be a measurable vector space, $X$ be a $V$-valued Gaussian random vector, $C \geq 1$, and let $g(\cdot)$ be a measurable $C$-quasi-additive functional on $V$. Then for any $\varepsilon>0$ there is a number $a_{0}>0$ (depending, generally speaking, on $X, g$, and $\varepsilon$ ) such that

$$
\mathrm{E} \exp \left\{a g^{b}(X)\right\}<\infty
$$

for all $a \in\left[0, a_{0}\right)$, where

$$
b=b(\varepsilon, C)=\frac{2}{1+(1+\varepsilon) \log _{2} C}
$$


Note that $b=2$ if $C=1$. Therefore Theorem 1 generalizes the result on the exponential integrability of measurable semiadditive functionals.

Inequality (1) holds for all $b \in[0, b(C))$ where

$$
b(C)=\frac{2}{1+\log _{2} C}
$$

and $C>1$. Note however that inequality (1) may hold with $b=b(C)$ for some measurable $C$-quasi-additive functionals.

Example 1. Let

$$
g(x)=p^{r}(x), \quad x \in V
$$

where $p(\cdot)$ is a measurable semiadditive functional and $r>1$. Then $g(\cdot)$ is a measurable $2^{r-1}$-quasi-additive functional, that is, $C=2^{r-1}$. Due to the theorem on the exponential integrability of semiadditive functionals, inequality (1) holds for $b=2 / r$. It remains to note that

$$
b=\frac{2}{r}=\frac{2}{1+\log _{2} 2^{r-1}}=\frac{2}{1+\log _{2} C} .
$$

Example 1 shows that not only the constant $b$ in inequality (1) can attain the value $b(C)$ but also that $b$ does not exceed $b(C)$ in the class of $C$-quasi-additive functionals. A natural question arises whether inequality (1) may hold for $b=b(C)$ in the class of quasi-additive functionals. The answer is positive under certain additional restrictions on the functional $g(\cdot)$.

Theorem 2. Let $(V, \mathcal{F})$ be a measurable vector space, $X$ be a $V$-valued Gaussian random vector, $C \in[1,2)$, and let $g(\cdot)$ be a measurable convex $C$-quasi-additive functional on $V$. Then there is a number $a_{0}>0$ (depending, generally speaking, on $X$ and $g$ ) such that

$$
\mathrm{E} \exp \left\{a g^{b}(X)\right\}<\infty
$$

for all $a \in\left[0, a_{0}\right)$, where

$$
b=\frac{2}{1+\log _{2} C} .
$$

An answer to the question on whether Theorem 2 holds for $C \geq 2$ requires an additional investigation.

\section{Proofs of the theorems}

We need several auxiliary results in order to prove Theorem 1.

Let $X_{1}, \ldots, X_{n}$ be independent copies of a $V$-valued Gaussian random vector $X$, where $n=2^{l}$ and $l \geq 1$. We introduce random vectors $S_{1}, \ldots, S_{n}$ as follows:

$$
\left(S_{1}, \ldots, S_{n}\right)^{\top}=U_{n}\left(X_{1}, \ldots, X_{n}\right)^{\top},
$$

where "T" denotes the transposition,

$$
U_{n}=n^{-1 / 2} H_{n}
$$

are real $n \times n$ matrices, and $H_{n}=\left(h_{k j}\right)_{k, j=1}^{n}$ are defined by

$$
H_{2}=\left(\begin{array}{cc}
1 & 1 \\
1 & -1
\end{array}\right), \quad H_{n}=\left(\begin{array}{cc}
H_{\frac{n}{2}} & H_{\frac{n}{2}} \\
H_{\frac{n}{2}} & -H_{\frac{n}{2}}
\end{array}\right), \quad n=2^{l}, l>1 .
$$

Therefore

$$
S_{k}=2^{-l / 2} \sum_{j=1}^{2^{l}} h_{k j} X_{j}
$$

for all $k=1, \ldots, 2^{l}$, where $h_{k j}= \pm 1$. 
Lemma 1 ([6]). The random vectors $S_{1}, \ldots, S_{n}$ are independent copies of the Gaussian random vector $X$.

Lemma 2. Let $C \geq 1$ and let $(g(x), x \in V)$ be a measurable $C$-quasi-additive functional. Then

$$
\begin{gathered}
g\left(2^{n} x\right) \leq(2 C)^{n} g(x), \\
g\left(x_{1}+\cdots+x_{n}\right) \leq C g\left(x_{1}\right)+\cdots+C^{n-2} g\left(x_{n-2}\right)+C^{n-1} g\left(x_{n-1}\right)+C^{n-1} g\left(x_{n}\right), \\
g\left(x_{1}+\cdots+x_{n}\right) \geq \frac{g\left(x_{1}\right)}{C}-C^{n-2} \sum_{k=2}^{n} g\left(-x_{k}\right)
\end{gathered}
$$

for all positive integers $n$ and all $x, x_{1}, \ldots, x_{n} \in V$.

Proof of Lemma 2. Inequalities (3) and (4) can be proved by induction. Inequality (5) follows from (4).

Lemma 3. Let $X$ be a $V$-valued Gaussian random vector, $C \geq 1$, and let $(g(x), x \in V)$ be a measurable $C$-quasi-additive functional on $V$. Then

$$
(\mathrm{P}\{g(X) \leq s\})^{4^{m}-1} \mathrm{P}\{g(X)>t\} \leq\left(\mathrm{P}\left\{g(X)>\frac{t-\left(4^{m}-1\right) C^{4^{m}-1} s}{C(2 C)^{m}}\right\}\right)^{4^{m}}
$$

for all positive integers $m$ and all $t>0$ and $s>0$.

Proof of Lemma 3. Let $X_{1}, \ldots, X_{4^{m}}$ be independent copies of $X$. In view of Lemma 1 , the random vectors

$$
S_{k}=2^{-m} \sum_{j=1}^{4^{m}} h_{k j} X_{j}, \quad k=1, \ldots, 4^{m},
$$

are independent copies of $X$.

Since $g(\cdot)$ is a $C$-quasi-additive symmetric functional, Lemma 2 implies

$$
\begin{aligned}
(2 C)^{m} g\left(S_{k}\right) & \geq g\left(2^{m} S_{k}\right)=g\left(\sum_{j=1}^{4^{m}} h_{k j} X_{j}\right) \\
& \geq \frac{1}{C} g\left(h_{k 1} X_{1}\right)-C^{4^{m}-2} \sum_{j=2}^{4^{m}} g\left(-h_{k j} X_{j}\right)=\frac{1}{C} g\left(X_{1}\right)-C^{4^{m}-2} \sum_{j=2}^{4^{m}} g\left(X_{j}\right)
\end{aligned}
$$

for all $k=1, \ldots, 4^{m}$. This yields

$$
\begin{aligned}
& (\mathrm{P}\{g(X) \leq s\})^{4^{m}-1} \mathrm{P}\{g(X)>t\}=\mathrm{P}\left\{g\left(X_{1}\right)>t, g\left(X_{2}\right) \leq s, \ldots, g\left(X_{4^{m}}\right) \leq s\right\} \\
& \quad \leq \mathrm{P}\left\{\frac{1}{C} g\left(X_{1}\right)-C^{4^{m}-2} \sum_{j=2}^{4^{m}} g\left(X_{j}\right)>\frac{t-\left(4^{m}-1\right) C^{4^{m}-1} s}{C}\right\} \\
& \quad \leq \mathrm{P}\left\{\bigcap_{k=1}^{4^{m}}\left\{g\left(S_{k}\right)>\frac{t-\left(4^{m}-1\right) C^{4^{m}-1} s}{C(2 C)^{m}}\right\}\right\} \\
& \quad=\left(\mathrm{P}\left\{g(X)>\frac{t-\left(4^{m}-1\right) C^{4^{m}-1} s}{C(2 C)^{m}}\right\}\right)^{4^{m}}
\end{aligned}
$$

for all $t>0$ and $s>0$.

Proof of Theorem 1. First we consider the case of a $C$-quasi-additive symmetric functional $g(\cdot)$. Consider a positive number $s=t_{0}$ such that

$$
c=\mathrm{P}\left\{g(X) \leq t_{0}\right\}>1 / 2 .
$$


For a given positive integer $m$ let the sequence of positive numbers $\left(t_{n}, n \geq 1\right)$ be defined as follows:

$$
t_{n}=\left((\gamma+1) \beta^{n}-\gamma\right) t_{0}, \quad n \geq 1,
$$

where

$$
\gamma=\frac{\alpha}{\beta-1}, \quad \alpha=\left(4^{m}-1\right) C^{4^{m}-1}, \quad \beta=C(2 C)^{m} \geq 2 .
$$

The sequence $\left(t_{n}, n \geq 1\right)$ is such that

$$
\frac{t_{n}-\alpha t_{0}}{\beta}=t_{n-1}, \quad n \geq 1,
$$

and $t_{n} \rightarrow \infty, n \rightarrow \infty$.

Let $z_{n}, n \geq 0$, be real numbers such that

$$
z_{0}=\frac{1-c}{c}, \quad c z_{n}=\mathrm{P}\left\{g(X)>t_{n}\right\}, \quad n \geq 1 .
$$

Note that $z_{0}<1$. It follows from (6) that $z_{n} \leq\left(z_{n-1}\right)^{4^{m}}, n \geq 1$. Thus

$$
\mathrm{P}\left\{g(X)>t_{n}\right\} \leq c\left(z_{0}\right)^{4^{m n}}, \quad n \geq 1,
$$

whence

$$
\begin{aligned}
E \exp \left\{a g^{b}(X)\right\} & \leq c\left[\exp \left\{a t_{0}^{b}\right\}+\sum_{n=1}^{\infty} z_{0}^{4^{m(n-1)}} \exp \left\{a\left(t_{0}(1+\gamma)\right)^{b} \beta^{b n}\right\}\right] \\
& =c\left[\exp \left\{a t_{0}^{b}\right\}+\sum_{n=1}^{\infty} z_{0}^{4^{m(n-1)}} \exp \left\{a\left(t_{0}(1+\gamma)\right)^{b} 2^{n b \log _{2} \beta}\right\}\right] \\
& =c\left[\exp \left\{a t_{0}^{b}\right\}+\sum_{n=1}^{\infty} z_{0}^{4^{m(n-1)}} \exp \left\{a\left(t_{0}(1+\gamma)\right)^{b} 4^{m n}\right\}\right] \\
& =c\left[\exp \left\{a t_{0}^{b}\right\}+\sum_{n=1}^{\infty} \exp \left\{4^{m n}\left[a\left(t_{0}(1+\gamma)\right)^{b}-\left(\left|\ln z_{0}\right| / 4^{m}\right)\right]\right\}\right]
\end{aligned}
$$

for

$$
b=b\left(\frac{1}{m}, C\right)=\frac{2}{1+\left(1+\frac{1}{m}\right) \log _{2} C} .
$$

This implies that the series converges and

$$
\mathrm{E} \exp \left\{a g^{b}(X)\right\}<\infty
$$

for $a<\left|\ln z_{0}\right| /\left[4^{m} a\left(t_{0}(1+\gamma)\right)^{b}\right]$. This completes the proof of the theorem for $C$-quasiadditive symmetric functionals $g(\cdot)$, since $m$ is arbitrary.

Now let $g(\cdot)$ be a measurable $C$-quasi-additive functional on $V$. Then

$$
\widetilde{g}(x)=\max \{g(x), g(-x)\}, \quad x \in V,
$$

is a measurable $C$-quasi-additive symmetric functional on $V$. Thus there is a number $a_{0}>0$ such that

$$
\mathrm{E} \exp \left\{a g^{b}(X)\right\} \leq \mathrm{E} \exp \left\{a \widetilde{g}^{b}(x)\right\}<\infty
$$

for all $a \in\left[0, a_{0}\right)$.

Remark 3. The proof of Theorem 1 is simpler for $C=1$. In this case, Theorem 1 follows from Lemma 3 for $m=1$ (see [6]).

The following result is needed for the proof of Theorem 2 . 
Lemma 4. Let $X$ be a $V$-valued Gaussian random vector, $C \in[1,2)$, and let $g(\cdot)$ be a measurable convex $C$-quasi-additive functional on $V$. If

$$
\mathrm{E} \exp \left\{a_{1} g(X)\right\}<\infty
$$

for some $a_{1}>0$, then there is $a>0$ such that

$$
\mathrm{E} \exp \left\{a g^{b}(X)\right\}<\infty
$$

where

$$
b=\frac{2}{1+\log _{2} C} .
$$

Proof of Lemma 4. It follows from Lemma 1 that

$$
\mathrm{P}\left\{g(X) \geq t 2^{s}\right\}=\mathrm{P}\left\{g\left(\frac{X_{1}+\cdots+X_{4^{n}}}{2^{n}}\right) \geq t 2^{s}\right\}
$$

for all positive integers $n$ and all $s, t>0$ where $X_{1}, \ldots, X_{4^{n}}$ are independent copies of the random vector $X$.

Since $g(\cdot)$ is a convex $C$-quasi-additive functional,

$$
\begin{aligned}
\mathrm{P}\left\{g\left(\frac{X_{1}+\cdots+X_{4^{n}}}{2^{n}}\right) \geq t 2^{s}\right\} & =\mathrm{P}\left\{g\left(\frac{2^{n}\left(X_{1}+\cdots+X_{4^{n}}\right)}{4^{n}}\right) \geq t 2^{s}\right\} \\
& \leq \mathrm{P}\left\{g\left(2^{n} X_{1}\right)+\cdots+g\left(2^{n} X_{4^{n}}\right) \geq t 2^{s+2 n}\right\} \\
& \leq \mathrm{P}\left\{g\left(X_{1}\right)+\cdots+g\left(X_{4^{n}}\right) \geq \frac{t 2^{s+n}}{C^{n}}\right\} .
\end{aligned}
$$

Condition (7) and the Markov inequality imply that

$$
\begin{aligned}
\mathrm{P}\left\{g(X) \geq t 2^{s}\right\} & \leq \mathrm{P}\left\{g\left(X_{1}\right)+\cdots+g\left(X_{4^{n}}\right) \geq \frac{t 2^{s+n}}{C^{n}}\right\} \\
& \leq \exp \left\{-a_{1} t \frac{2^{s+n}}{C^{n}}\right\} \mathrm{E} \exp \left\{a_{1}\left(g\left(X_{1}\right)+\cdots+g\left(X_{4^{n}}\right)\right)\right\} \\
& =\exp \left\{-a_{1} t \frac{2^{s+n}}{C^{n}}\right\}\left(\mathrm{E} \exp \left\{a_{1} g(X)\right\}\right)^{4^{n}}=\exp \left\{-a_{1} t \frac{2^{s+n}}{C^{n}}+a_{2} 4^{n}\right\}
\end{aligned}
$$

for all positive integers $n$ and all $s, t>0$ where $a_{2}=\ln \mathrm{E} \exp \left\{a_{1} g(X)\right\} \in(0, \infty)$. In the case of $s=n\left(1+\log _{2} C\right)=2 n / b$ one has

$$
\mathrm{P}\left\{g(X) \geq t 4^{n / b}\right\} \leq \exp \left\{-\left(a_{1} t-a_{2}\right) 4^{n}\right\}
$$

for all positive integers $n$ and all $t>0$. This implies that

$$
\mathrm{E} \exp \left\{a g^{b}(X)\right\} \leq \exp \left\{4 a t^{b}\right\}+\sum_{n=1}^{\infty} \exp \left\{-\left[a_{1} t-4 a t^{b}-a_{2}\right] 4^{n}\right\} .
$$

The series converges and

$$
\mathrm{E} \exp \left\{a g^{b}(X)\right\}<\infty
$$

if $t>a_{2} / a_{1}$ and $0 \leq a<\left(a_{1} t-a_{2}\right) / 4 t^{b}$.

Proof of Theorem 2. Theorem 2 for $C=1$ follows from Theorem 1 immediately. Condition (7) holds by Theorem 1 if $C \in(1,2)$, in which case one needs to apply Lemma 4. 


\section{BIBLIOGRAPHY}

1. V. P. Skitovich, Linear forms of independent variables and the normal law of distribution, Izv. Akad. Nauk SSSR, Ser. Mat. 18 (1954), 952. (Russian) MR 16:52a

2. N. N. Vakhania, V. I. Tarieladze, and S. A. Chobanyan, Probability Distributions on Banach Spaces, "Nauka", Moscow, 1985; English transl., Kluwer, Dordrecht, 1987. MR 86j:60014; MR 97k:60007

3. A. V. Skorokhod, A remark on Gaussian measures in a Banach space, Teor. Veroyatnost. i Primenen. 15 (1970), no. 3, 519-520; English transl., Theory Probab. Appl. 15 (1971), 508509. MR 43:3417

4. X. Fernique, Intégrabilité des vecteurs gaussiens, C. R. Acad. Sci. Paris Sér. I. Math. 270 (1970), no. 7, 1698-1699. MR 42:1170

5. H. J. Landau and L. A. Shepp, On the supremum of a Gaussian process, Sankhya 32 (1970), no. 4, 369-378. MR 44:3381

6. V. V. Buldygin and D. M. Severnyuk, On the exponential integrability of semiadditive functionals of Gaussian vectors Teor. Imovirnost. Matem. Statist. 60 (1999), 11-16; English transl., Theory Probab. Math. Statist. 60 (2000), 13-18.

Department of Analysis and Probability Theory, National Technical University of Ukraine (KPI), Prospekt Peremogy 37, Kyiv-56 02056, Ukraine

E-mail address: valbuld@comsys.ntu-kpi.kiev.ua

Received 13/DEC/2002

Translated by OLEG KLESOV 